\title{
Institutional Review Board Roles and Functions to Conduct Clinical Trials at a Medium-Sized Regional Hospital, Japan
}

\author{
Maki Ogawa ${ }^{1}$, Rie Matsumoto ${ }^{2}$ and Atsushi Imai ${ }^{3 *}$ \\ ${ }^{1}$ Clerk Support Unit for Physicians, Japan \\ ${ }^{2}$ Pharmaceutical Department, Japan \\ ${ }^{3}$ Institute of Endocrine-related Cancer, Matsunami General Hospital, Japan
}

Received: December 21, 2017; Published: January 05, 2018

*Corresponding author: Atsushi Imai, Institute of Endocrine-related Cancer, Matsunami General Hospital, Kasamatsu, Gifu, Japan.

Key words: IRB; Contracted Clinical Trial; Medical Institution Anchor of the Region; Medical Research

\section{Introduction}

Clinical trials are a key resource for the discovery of new diagnostic, treatment and prevention methods for disease. Many of today's most introduced prevention and treatment modalities are based on previous clinical trial results. Clinical trials are an important part of improving quality of health care [1]. Facilitators of participation in clinical trials have been linked to recommendations by reliable health care providers, a supportive family, satisfactory doctor-patient relationship that depends on culture, financial incentives, anticipation of a personal benefit to participants, convenience to participants and culturally appropriate advertisements [2]. To address such facilitators and barriers of clinical trial participation in communities, we report our institutional review board (IRB) of Matsunami General Hospital [3]. A typical central medical institution anchor of the region, Japan.

\section{Role of IRB}

IRB has the authority for jurisdiction over research involving primarily drugs, devices and biology's that must be approved by the Ministry of Health, Labor and Welfare, Japan. The clinical research or trials are defined as: "research conducted on human subjects to determine or verify pharmacologic, absorption, metabolism, safety and efficacy of one or more research products, in addition to clinical or pharmacodynamics effects and adverse events or reactions."

Table 1: Contracted clinical research 2004-2016.

\section{IRB Members}

We do not include a member of more than one ethic committee and managers of the institution in which the trial is going to be concluded. IRB consists of following background members:

a) 3 medical experts with experience in international study designed in compliance with good clinical practice

b) 1 nurse who has over 20-year experience in clinical practice

c) 1 pharmacist who has over 20-year experience in clinical practice

d) 3 non-medical persons

e) 2 persons with learned knowledge and experience who are unaffiliated with the institution

f) 2 persons in clerical support unit

\section{Current statue of Clinical Trial Acceptance}

A total of 32 clinical trials were completed in our institution between 2004 and 2016 (Table 1). Research subjects were distributed different disciplines, approximately $30 \%$ being diabetes mellitus-related.

\begin{tabular}{|l|c|c|}
\hline Category & Target illness & Number of clinical trials \\
\hline \multirow{3}{*}{ Metabolism } & Diabetes Mellitus & 10 \\
\cline { 2 - 3 } & Diabetic neuropathy & 1 \\
\cline { 2 - 3 } & Diabetic neuropathy & 1 \\
\hline \multirow{2}{*}{ Respiratory } & Asthma & 2 \\
\cline { 2 - 3 } & Influenza & 3 \\
\hline
\end{tabular}




\begin{tabular}{|c|c|c|}
\hline \multirow{2}{*}{$\begin{array}{c}\text { Hepatology } \\
\text { Reumatology }\end{array}$} & Liver cirrhosis & 1 \\
\cline { 2 - 3 } & Knee ostheoarthritis & 1 \\
\cline { 2 - 3 } Digestive tube & Reumatoid arthritis & 1 \\
\hline \multirow{2}{*}{ Cerebrovascular disease } & Gastric ulcer & 1 \\
\cline { 2 - 3 } & Crohn's disease & 2 \\
\hline \multirow{2}{*}{ Circulation } & Gastroesophageal reflux disease & 3 \\
\hline & Ischemic stroke & 2 \\
\cline { 2 - 3 } & Hypertension & 2 \\
\hline
\end{tabular}

\section{Comments}

Clinical trials accepted in our institution are in quality and quantity inferior as compared with those in government institutions of similar size [4]. One of major differences may result from the provoked evolution of regulations. Our institution regulations on clinical trials may be structured with the aim of attracting more community-based trials to the regional community and to meet the criteria of management policies. Aside from the institutional incentives, the lack of accreditation of IRBs raises concerns regarding the compliance of organizations, IRBs or the researchers and/or the research staff with a human study protection attempt, quality assurance and quality improvement measure accountability, adequate resources availability, and transparency by communication and interaction with the community, research participants and researchers. Encouraging IRBs to apply for accreditation would strengthen any flaws in structure of operating protocols and help our institutional circumstance to have its goal to raise its share in drug research investments. Before approving the trials, IRBs should introduce the policy to better assess the existing uncertainties in a given investigation study, employing tools, such as survey of specialists, official announcement of the protocols and systematic reviews with a focus on identifying golden standard treatment that should be contained in consideration of the proposed trial, as previously described [5]. It may be the most plausible to make an assertion that the IRB needs contributing to improvement in quality and quantity of clinical trials.

\section{References}

1. Ekmekci PE (2016) Main ethical breaches in multicenter clinical trials regulations of turkey. Med Law 35: 491-508.

2. Ma G, Tan Y, Blakeney N, Seals B, Ma X, et al. (2014) The impact of a community-based clinical trial educational intervention among underrepresented chinese americans. Cancer Epidemiol Biomarkers Prev 23: 424-432.

3. Imai A, Kajikawa H, Koiwai C, Hiroshi Takagiand, Ichigo S (2018) Great role in gynecological cancer prophylaxis of a unique health check-up institute, ningen dock in japan. In: R Rajkumar (Eds.), Cervical Cancer Screening, Treatment and Prevention, In Teck, Rijeka, Croatia.

4. Ito T (2016) Differences in investigator-initiated trials between japan and other countries: Analyses of clinical trials sponsored by academia and government in the clinicaltrials.Gov registry and in the three japanese registries. PLoS One 11: e0148455.

5. Mann H, Djulbegovic B (2003) Choosing a control intervention for a randomised clinical trial. BMC Med Res Methodol p 3:7.

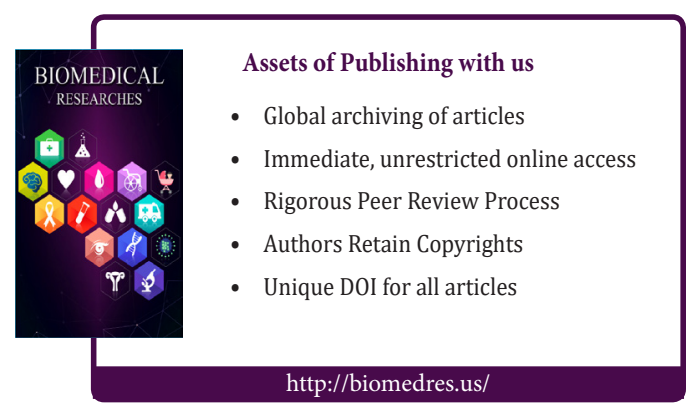

